\title{
On a universal axiomatization of the real closed fields
}

\author{
by KRzYszTof Jan NowaK (Kraków and Amsterdam)
}

\begin{abstract}
This paper presents a natural axiomatization of the real closed fields. It is universal and admits quantifier elimination.

1. Introduction. In model theory there are two ways to reduce axioms of a theory $T$ only to some patterns for blocks of quantifiers, as well as to emphasize the set of quantifier-free formulae (through elimination) and the set of existential formulae (by model completeness). They both consist in adding extra symbols; namely, Skolem relations in order to obtain the Morley expansion of $T$, and Skolem functions to construct the iterated Skolem expansion of $T$ (cf. [6]). Whereas the former expansion is inductive (i.e. has axioms of the form $\forall \exists \phi$ ) and admits quantifier elimination, the latter is universal (i.e. has axioms of the form $\forall \phi$ ) and model complete.

The theory of real closed fields is inductive (which is very frequent within abstract algebra) and admits quantifier elimination (due to Tarski [10]; also cf. [3] or [4]). In Section 2 we expand the language of ordered fields by adding new function symbols for each global Nash function and for all arithmetic roots. In this fashion we are able to formulate some natural, universal postulates which turn out to admit quantifier elimination. In order to prove that those universal axioms describe exactly the real closed fields containing $\mathbb{R}$, we make use of valuation theory and Hensel's lemma for Nash germs. Finally, Section 3 illustrates the above by an application to a problem of definability concerning semialgebraic functions.

A broad exposition of semialgebraic geometry, including the theory of Nash functions and sets, is presented in [3]. For the essentials of model
\end{abstract}

1991 Mathematics Subject Classification: 03C10, 12D15, 14P20.

Key words and phrases: real closed fields, real valuations, Nash functions, quantifier elimination.

Research partially supported by Grant no. 2 P03A 06108. 
theory, we refer the reader to e.g. [4] and [6]. A sufficient elaboration of valuation theory can be found in [8] and [9].

2. Universal axioms for the real closed fields. We first set up basic notation and terminology concerning field theory (cf. [3], [4] or [8]). For simplicity, we shall omit in the sequel the initial quantifiers in universal formulae.

An ordering of a field $K$ is an order relation which satisfies the two postulates

$$
\begin{gathered}
x \leq y \Rightarrow x+z \leq y+z, \\
0 \leq x \wedge 0 \leq y \Rightarrow 0 \leq x y .
\end{gathered}
$$

A positive cone of $K$ is a subset $P$ of $K$ satisfying

$$
P+P \subseteq P, \quad P \cdot P \subseteq P, \quad P \cap-P=\{0\}, \quad P \cup-P=K .
$$

There is a one-to-one correspondence between the orderings of the field $K$ and the positive cones of $K$ :

$\leq$ defines a positive cone $P:=\{a \in K: 0 \leq a\}$

$P$ defines an ordering $a \leq b \Leftrightarrow b-a \in P$.

A field $K$ is said to be real if it satisfies one of the following equivalent conditions:

(i) $K$ can be ordered;

(ii) $-1 \notin \sum K^{2}$;

(iii) $\sum x_{i}^{2}=0 \Rightarrow$ all $x_{i}=0$;

(iv) $\sum K^{2} \neq K$.

Here $\sum K^{2}$ stands for the set of sums of squares of $K$.

An ordered field $(K, \leq)$ is called maximally ordered if it has no proper algebraic ordered field extension $\left(K^{\prime}, \leq^{\prime}\right)$. Every positive element of a maximally ordered field $K$ is a square, whence $K$ has a unique ordering.

A field $K$ is called real closed if it is real, but has no real proper algebraic field extension $K^{\prime}$. A field $K$ is real closed iff it has a unique ordering and is maximally ordered.

Within the language $\{+,-, \cdot 1 /, 0,1\}$, the theory of fields can be universally axiomatized in the ordinary fashion. Here,,$+- \cdot$ denote the obvious binary function symbols, and $1 /$ is a unary function symbol characterized by the postulate

$$
(x \neq 0 \Rightarrow x \cdot 1 / x=1) \wedge(x=0 \Rightarrow 1 / x=0) .
$$

The standard axiomatization of the theory of real closed fields is based on the following Artin-Schreier theorem [1]: 
For a field $K$, the three conditions are equivalent:

(i) $K$ is real closed;

(ii) $K^{2}$ is a positive cone of $K$ and every polynomial of odd degree has a root in $K$;

(iii) $K[i]=K[X] /\left(X^{2}+1\right)$ is an algebraically closed field.

The real closed fields are thus described by the axioms of ordered field theory augmented by the following ones:

$$
\begin{gathered}
\forall x \exists y\left(0 \leq x \Rightarrow x=y^{2}\right) \\
\forall x_{1}, \ldots, x_{2 n-1} \exists y y^{2 n-1}+x_{1} y^{2 n-2}+\ldots+x_{2 n-1}=0(n=1,2, \ldots) .
\end{gathered}
$$

Now we shall attach a collection of function symbols to the language of ordered fields $\{\leq,+,-, \cdot, 1 /, 0,1\}$. First we add the symbols of arithmetic roots $\sqrt[n]{ }(n=2,3, \ldots)$ characterized by the postulates

$$
\left(0 \leq x \Rightarrow 0 \leq \sqrt[n]{x} \wedge(\sqrt[n]{x})^{n}=x\right) \wedge(x \leq 0 \Rightarrow \sqrt[n]{x}=0)
$$

Next, for each global Nash function $f: \mathbb{R}^{n} \rightarrow \mathbb{R}$, we pick up one of the quantifier-free formulae $\phi_{f}\left(x_{1}, \ldots, x_{n}, y\right)$ that defines the graph of $f$, and add the $n$-ary function symbol $f^{\&}$ subject to the postulates

$$
\begin{gathered}
\phi_{f}\left(x_{1}, \ldots, x_{n}, f^{\&}\left(x_{1}, \ldots, x_{n}\right)\right), \\
(f+g)^{\&}\left(x_{1}, \ldots, x_{n}\right)=f^{\&}\left(x_{1}, \ldots, x_{n}\right)+g^{\&}\left(x_{1}, \ldots, x_{n}\right), \\
(f \cdot g)^{\&}\left(x_{1}, \ldots, x_{n}\right)=f^{\&}\left(x_{1}, \ldots, x_{n}\right) \cdot g^{\&}\left(x_{1}, \ldots, x_{n}\right) .
\end{gathered}
$$

Let $\mathcal{L}$ denote the language of ordered fields expanded in this way, and let $T$ be the theory of $\mathcal{L}$ specified by the axioms of ordered fields augmented by the ones describing the arithmetic roots and global Nash functions. Given a model $K^{\&}$ of $T$, denote by $f^{K}$ the interpretation of each function symbol $f^{\&}$ of $\mathcal{L}$. Clearly, every real number $a$ determines the constant symbol $a^{\&}$ of $\mathcal{L}$, and the map

$$
\mathbb{R} \rightarrow K, \quad a \mapsto a^{K},
$$

is an ordered field embedding. From now on, we shall identify $\mathbb{R}$ with its image under this embedding.

By virtue of the Tarski-Seidenberg transfer principle, every real closed field $\bar{K}$ containing $\mathrm{R}$ is the reduct of a unique model $\bar{K}^{\&}$ of $T$. Indeed, one must construe each function symbol $f^{\&}$ as a unique extension of $f$ from $\mathrm{R}$ to the field $\bar{K}$, which is defined by the formula $\phi_{f}$ (cf. [3], Chap. 5).

Since every ordered field $K$ can be embedded in a real closed field $\bar{K}$, it follows that - for any model $K^{\&}$ of $T$ and any $n$-ary function symbol $f^{\&}$ of $\mathcal{L}$ - the sole interpretation of $f^{\&}$ in $K^{\&}$ is

$$
f^{K}=\text { restriction of } f^{\bar{K}} \text { to } K^{n} \text {. }
$$


Consequently, $f^{K}$ does not depend on the formula $\phi_{f}$ chosen at the beginning. The following basic rules are therefore satisfied:

1) If $X_{i}$ denotes the ith coordinate projection $(i=1, \ldots, n)$, then

$$
X_{i}^{\&}\left(x_{1}, \ldots, x_{n}\right)=x_{i} .
$$

2) If $g^{\&}$ is an $n$-ary and all $f_{i}^{\&}$ are $m$-ary symbols of $\mathcal{L}$, then

$$
\begin{aligned}
g\left(f_{1}\left(x_{1}, \ldots, x_{m}\right), \ldots,\right. & \left.f_{n}\left(x_{1}, \ldots, x_{m}\right)\right)^{\&} \\
& =g^{\&}\left(f_{1}^{\&}\left(x_{1}, \ldots, x_{m}\right), \ldots, f_{n}^{\&}\left(x_{1}, \ldots, x_{m}\right)\right) .
\end{aligned}
$$

3) For any Nash function $f: \mathbb{R}^{n} \rightarrow \mathbb{R}$ and $a \in \mathbb{R}^{n}$, we have

$$
f^{K}(a)=f(a) \text {. }
$$

4) For any Nash functions $f, g: \mathbb{R}^{n} \rightarrow \mathbb{R}$, we have $f^{K}<g^{K}$ (respectively $f^{K} \leq g^{K}$ ) whenever $f<g$ (respectively $f \leq g$ ).

We shall now prove that every model $K^{\&}$ of $T$ is a real closed field. The chief tool we use is the theory of henselian valued fields. Therefore, we recall without proofs some of the basic facts on valued fields (cf. [8] or [9]). Let $(G, \leq)$ be an ordered abelian group written additively. A map $v: K \rightarrow G \cup\{\infty\}$ is said to be a valuation of a field $K$ if it satisfies the conditions

$$
\begin{gathered}
v(x)=\infty \Leftrightarrow x=0, \\
v(x \cdot y)=v(x)+v(y), \quad v(x+y) \geq \min \{v(x), v(y)\},
\end{gathered}
$$

where $\infty$ is greater than any element $g$ of $G$, and

$$
g+\infty=\infty+g=\infty .
$$

It is easy to see that

$$
\begin{gathered}
v(1)=0, \quad v(-x)=v(x), \\
v(x) \neq v(y) \Rightarrow v(x+y)=\min \{v(x), v(y)\} .
\end{gathered}
$$

The pair $(K, v)$ is called a valued field.

By a valuation ring of a field $K$ we mean a subring $\mathfrak{R}$ of $K$ such that if $a \notin \mathfrak{R}$, then $1 / a \in \mathfrak{R}$. $\mathfrak{R}$ is a local ring; we introduce the following notation:

$\mathfrak{M}=$ the sole maximal ideal of $\mathfrak{R}$,

$U=$ the set of all units in $\mathfrak{R}$,

$F=\mathfrak{R} / \mathfrak{M}$, the residue class field of $\mathfrak{R}$.

There is a one-to-one correspondence between the valuation rings of a field $K$ and the valuations of $K$ (up to equivalence of valuations). A valuation $v$ determines a valuation ring

$$
\mathfrak{R}=\mathfrak{R}_{v}:=\{a \in K: v(a) \geq 0\} ;
$$


then

$$
\mathfrak{M}=\mathfrak{M}_{v}=\{a \in K: v(a)>0\}, \quad U=U_{v}=\{a \in K: v(a)=0\},
$$

and $F=F_{v}=\mathfrak{R} / \mathfrak{M}$ is called the residue class field of $v$.

Conversely, a valuation ring $\mathfrak{R}$ of $K$ induces a group

$$
G:=(K \backslash\{0\}) / U
$$

with additively written group structure generated by multiplication in $K \backslash$ $\{0\}$; let $v: K \backslash\{0\} \rightarrow G$ be the canonical map. We define an ordering on $G$ as follows:

$$
v(a) \leq v(b) \Leftrightarrow b a^{-1} \in \Re_{v} .
$$

A valuation $v$ of a field $K$ is called real if its residue class field $F_{v}$ is real. A valuation $v$ is called henselian if its valuation ring $\mathfrak{R}_{v}$ is henselian.

We say that an ordering $\leq$ of a field $K$ and a valuation $v$ of $K$ are compatible if

$$
0<x \leq y \Rightarrow v(x) \geq v(y)
$$

For every real valuation $v$ of $K$, there exists an ordering of $K$ compatible with $v$. In particular, a field $K$ is real whenever it has a real valuation.

Every ordered field $(K, \leq)$ determines the valuation ring $\mathfrak{R}_{K}$ of all elements of $K$ bounded in absolute value by a rational number; its maximal ideal $\mathfrak{M}_{K}$ consists of all infinitesimals of $K$. The ring $\mathfrak{R}=\mathfrak{R}_{K}$ induces a valuation $v=v_{K}$ compatible with the ordering $\leq$ of $K$, and called the standard valuation of $(K, \leq)$.

A key role in the proof we proceed with will be played by the criterion below (cf. [8], p. 87):

CRITERION. A necessary and sufficient condition for a real valued field $(K, v)$ to be real closed is that its residue class field $F_{v}$ be real closed, its valuation ring $\mathfrak{R}_{v}$ be henselian and the valuation group $G$ be divisible.

We now state an elementary lemma which can be checked by straightforward computation.

LEMMA 1. For a real number $\delta>0$, consider the two functions of one variable:

$$
g_{\delta}:(-\delta, \delta) \rightarrow \mathbb{R}, \quad y=g_{\delta}(x)=\frac{2 \delta x}{\delta^{2}-x^{2}}
$$

and

$$
h_{\delta}: \mathbb{R} \rightarrow(-\delta, \delta), \quad x=h_{\delta}(y)=\frac{\delta y}{\sqrt{y^{2}+1}+1},
$$

as well as their counterparts over a model $K^{\&}$ of the theory $T$ :

$$
g_{\delta}^{K}:(-\delta, \delta) \rightarrow K \quad \text { and } \quad h_{\delta}^{K}: K \rightarrow(-\delta, \delta),
$$


defined by the same formulae. Then the functions $g_{\delta}$ and $h_{\delta}$ as well as $g_{\delta}^{K}$ and $h_{\delta}^{K}$ are mutually inverse. The function $h_{\delta}^{K}$ is the one represented by the symbol $h_{\delta}^{\&}$.

Lemma 2. Let $f: \mathbb{R}^{n} \rightarrow \mathbb{R}$ be a Nash function, $K^{\&}$ be a model of $T$, and let $v$ be the standard valuation of $K$ with valuation ring $(\mathfrak{R}, \mathfrak{M})$. Then for all $\left(\lambda_{1}, \ldots, \lambda_{n}\right) \in \mathfrak{M}^{n}$ we have

$$
f^{K}\left(\lambda_{1}, \ldots, \lambda_{n}\right) \in f(0)+\mathfrak{M} \subset \mathfrak{R}
$$

For, let $\varepsilon>0$ be any positive real number, and pick up a real number $\delta>0$ such that

$$
f\left(a_{1}, \ldots, a_{n}\right)<f(0)+\varepsilon \quad \text { for all } a_{i} \in \mathbb{R}, \quad a_{i} \in(-\delta, \delta) .
$$

Then $f \circ h_{\delta}<f(0)+\varepsilon$, whence $f^{K} \circ h_{\delta}^{K}<f(0)+\varepsilon$. Since each infinitesimal $\lambda_{i} \in(-\delta, \delta)$, we have

$f^{K}\left(\lambda_{1}, \ldots, \lambda_{n}\right)=f^{K} \circ\left(h_{\delta}^{K}, \ldots, h_{\delta}^{K}\right) \circ\left(g_{\delta}^{K}, \ldots, g_{\delta}^{K}\right)\left(\lambda_{1}, \ldots, \lambda_{n}\right)<f(0)+\varepsilon$.

Similarly, $f(0)-\varepsilon<f^{K}\left(\lambda_{1}, \ldots, \lambda_{n}\right)$, and thus (as $\varepsilon>0$ has been chosen arbitrarily small) $f^{K}\left(\lambda_{1}, \ldots, \lambda_{n}\right) \in f(0)+\mathfrak{M}$.

Proposition. Let $K^{\&}$ be a model of $T$. Then the standard valuation $v$ of $K$ is henselian.

Our proposition asserts that the valuation $\operatorname{ring}(\mathfrak{R}, \mathfrak{M})$ of the standard valuation $v$ of $K$ is henselian. Take any monic polynomial

$$
p(T)=T^{n}+\alpha_{1} T^{n-1}+\ldots+\alpha_{n} \in \mathfrak{R}[T] .
$$

As the field $K$ contains $\mathbb{R}$, the residue class field $F$ of $v$ is exactly $\mathbb{R}$. Hence

$$
\alpha_{i}=a_{i}+\lambda_{i} \quad \text { for some } a_{i} \in \mathbb{R}, \quad \lambda_{i} \in \mathfrak{M}(i=1, \ldots, n) .
$$

Supposing that $t \in \mathbb{R}$ is a simple root of the reduced polynomial

$$
\bar{p}(T)=T^{n}+a_{1} T^{n-1}+\ldots+a_{n} \in \mathbb{R}[T],
$$

we must find a root $\vartheta$ of the polynomial $p(T)$ in the set $t+\mathfrak{M}$.

Consider the polynomial

$$
T^{n}+\left(a_{1}+x_{1}\right) T^{n-1}+\ldots+\left(a_{n}+x_{n}\right),
$$

where $x_{1}, \ldots, x_{n}$ are indeterminates. It follows from Hensel's lemma that there exists a Nash germ $f\left(x_{1}, \ldots, x_{n}\right)$ at $0 \in \mathbb{R}^{n}$ for which $f(0)=t$ and

$$
f^{n}\left(x_{1}, \ldots, x_{n}\right)+\left(a_{1}+x_{1}\right) f^{n-1}\left(x_{1}, \ldots, x_{n}\right)+\ldots+\left(a_{n}+x_{n}\right)=0 .
$$

We may assume that $f$ is defined on a cube $(-\delta, \delta)^{n}, \delta>0$, so that $f \circ$ $\left(h_{\delta}, \ldots, h_{\delta}\right)$ is a global Nash function. Then

$$
\begin{array}{r}
f^{n}\left(h_{\delta}\left(x_{1}\right), \ldots, h_{\delta}\left(x_{n}\right)\right)+\left(a_{1}+h_{\delta}\left(x_{1}\right)\right) \cdot f^{n-1}\left(h_{\delta}\left(x_{1}\right), \ldots, h_{\delta}\left(x_{n}\right)\right) \\
+\ldots+\left(a_{n}+h_{\delta}\left(x_{n}\right)\right)=0,
\end{array}
$$


and thus

$$
\begin{aligned}
& \left(f^{n} \circ\left(h_{\delta}, \ldots, h_{\delta}\right)\right)^{K}\left(x_{1}, \ldots, x_{n}\right) \\
& \quad+\left(a_{1}+h_{\delta}^{K}\left(x_{1}\right)\right) \cdot\left(f^{n-1} \circ\left(h_{\delta}, \ldots, h_{\delta}\right)\right)^{K}\left(x_{1}, \ldots, x_{n}\right) \\
& \quad+\ldots+\left(a_{n}+h_{\delta}^{K}\left(x_{n}\right)\right)=0 .
\end{aligned}
$$

Hence, replacing $x_{i}$ by $g_{\delta}^{K}\left(\lambda_{i}\right)$, we get

$$
\begin{aligned}
& \left(f^{n} \circ\left(h_{\delta}, \ldots, h_{\delta}\right)\right)^{K}\left(g_{\delta}^{K}\left(\lambda_{1}\right), \ldots, g_{\delta}^{K}\left(\lambda_{n}\right)\right) \\
& +\left(a_{1}+\lambda_{1}\right) \cdot\left(f^{n-1} \circ\left(h_{\delta}, \ldots, h_{\delta}\right)\right)^{K}\left(g_{\delta}^{K}\left(\lambda_{1}\right), \ldots, g_{\delta}^{K}\left(\lambda_{n}\right)\right) \\
& +\ldots+\left(a_{n}+\lambda_{n}\right)=0 .
\end{aligned}
$$

This signifies that

$$
\vartheta:=\left(f \circ\left(h_{\delta}, \ldots, h_{\delta}\right)\right)^{K}\left(g_{\delta}^{K}\left(\lambda_{1}\right), \ldots, g_{\delta}^{K}\left(\lambda_{n}\right)\right)
$$

is a root of the polynomial $p(T)$. From Lemma 2 it follows that

$$
\vartheta \in\left(f \circ\left(h_{\delta}, \ldots, h_{\delta}\right)\right)(0)+\mathfrak{M}=f(0)+\mathfrak{M}=t+\mathfrak{M},
$$

whereby the proof is completed.

Since each positive element $x$ of $K^{\&}$ has all roots, the group of the standard valuation $v$ of $K$ is divisible. The residue class field of $v$ is $\mathbb{R}$ because $\mathbb{R}$ is the maximal archimedian ordered subfield of $K$. Consequently, the above proposition together with the criterion for a real valued field to be real closed imply

Corollary. Every model $K^{\&}$ of the theory $T$ is a real closed field.

Finally, observe that using the axioms of $T$, one can replace any formula with function symbols $f^{\&}$ by a formula of the language of ordered fields, and thus quantifier elimination within the theory of real closed fields is applicable to the theory $T$. Summing up, we can therefore state the following

MAIN THEOREM. The universal theory $T$ admits quantifier elimination. The assignment to each model $K^{\&}$ of its reduct $K$ is a one-to-one correspondence between the models of $T$ and the real closed fields containing $\mathbb{R}$.

3. An application to semialgebraic geometry. Universal theories are characterized by the preservation theorem below, due to Tarski [11] and Łoś [7] (also cf. [4], Chap. 3 or [6], Sect. 3):

A theory $T$ has a set of universal axioms iff it is preserved under submodels.

We shall now demonstrate how this preservation property of our theory $T$ of real closed fields applies to problems concerning semialgebraic functions.

Proposition. Each semialgebraic function $f: \mathbb{R}^{n} \rightarrow \mathbb{R}$ is piecewise defined by a finite number of $\mathcal{L}$-terms 


$$
\tau_{1}\left(x_{1}, \ldots, x_{n}\right), \ldots, \tau_{s}\left(x_{1}, \ldots, x_{n}\right),
$$

i.e., for all $a=\left(a_{1}, \ldots, a_{n}\right) \in \mathbb{R}^{n}, f(a)=\tau_{i}(a)$ for some $i=1, \ldots, s$.

The proof is by reductio ad absurdum. Consider a quantifier-free formula $\phi\left(x_{1}, \ldots, x_{n}, y\right)$ that defines the graph of $f$, and the type

$\Sigma\left(x_{1}, \ldots, x_{n}\right)=\left\{\neg \phi\left(x_{1}, \ldots, x_{n}, \tau\left(x_{1}, \ldots, x_{n}\right)\right): \tau\right.$ is a term of $\left.\mathcal{L}\right\}$.

Supposing $f$ is not piecewise defined by a finite number of terms, it follows from the compactness theorem (cf. [4], Chap. 2) that the type $\Sigma$ is consistent with the theory $T$. In other words, $T$ has a model $K$ which realizes $\Sigma$, i.e. some $n$-tuple $\left(a_{1}, \ldots, a_{n}\right)$ of elements of $K$ satisfies $\Sigma$ in $K$. The submodel $M$ generated by $\left\{a_{1}, \ldots, a_{n}\right\}$ is of the form

$$
M=\left\{\tau\left[a_{1}, \ldots, a_{n}\right]: \tau \text { is a term of } \mathcal{L}\right\} .
$$

As the theory $T$ is universal, $M$ is its model too. Through quantifier elimination, $M$ is an elementary submodel of $K: M \prec K$. But $M$ models the sentence

$$
\forall y \neg \phi\left(a_{1}, \ldots, a_{n}, y\right),
$$

which is not true in $K$. We have thus obtained a contradiction with $M \prec K$.

Corollary. Every semialgebraic function $f: \mathbb{R}^{n} \rightarrow \mathbb{R}$ is piecewise Nash. In other words, there exists a disjoint decomposition of $\mathbb{R}^{n}$ into finitely many Nash submanifolds $M_{k}(k=1, \ldots, s)$ such that the restriction of $f$ to each $M_{k}$ is a Nash function. Moreover, we can assume each $M_{k}$ to be Nash diffeomorphic to the unit cube $(0,1)^{\operatorname{dim} M_{k}}$.

Indeed, every semialgebraic set is a disjoint union of finitely many Nash submanifolds $M_{k}$; moreover, we can assume each $M_{k}$ to be Nash diffeomorphic to the unit cube $(0,1)^{\operatorname{dim} M_{k}}$ (cf. [3], Prop. 8.1.12). In view of the above proposition, we may therefore limit ourselves to the case when $f$ is defined by one $\mathcal{L}$-term $\tau$. Now, the assertion follows easily by induction with respect to the complexity of the term $\tau$ (which is built from roots and global Nash functions by means of four arithmetic operations,,$+- \cdot$ and $1 /$ ). We need again to decompose semialgebraic sets into Nash submanifolds.

R e mark. The above corollary can also be obtained via ordinary apparatus of semialgebraic geometry. It extends to the domain of semianalytic geometry provided that $f$ is a bounded semianalytic function. Whereas the geometric proof makes use of a partition technique applied to the graph of a given semialgebraic or semianalytic function $f$ (cf. [5] or [2], Sect. 3), the arguments we have presented illustrate some general methods of model theory. 
Acknowledgements. This article was written during the author's stay at the Vrije Universiteit in Amsterdam. The author thanks Professor Jacek Bochnak from the Vrije Universiteit for his invitation and hospitality.

\section{References}

[1] E. Artin und O. Schreier, Algebraische Konstruktion reeller Körper, Abh. Math. Sem. Univ. Hamburg 5 (1927), 85-99.

[2] E. Bierstone and P. D. Milman, Semianalytic and subanalytic sets, Publ. Math. I.H.E.S. 67 (1988), 1-42.

[3] J. Bochnak, M. Coste and M.-F. Roy, Géométrie Algébrique Réelle, Springer, 1987.

[4] C. C. Chang and H. J. Keisler, Model Theory, North-Holland, Amsterdam, 1973.

[5] Z. Denkowska, S. Łojasiewicz et J. Stasica, Certaines propriétés élémentaires des ensembles sous-analytiques, Bull. Acad. Polon. Sci. Sér. Sci. Math. 27 (1979), 529-536.

[6] H. J. Keisler, Fundamentals of model theory, in: Handbook of Mathematical Logic, North-Holland, Amsterdam, 1977, 47-103.

[7] J. Łoś, On the extending of models I, Fund. Math. 42 (1955), 38-54.

[8] A. Prestel, Lectures on Formally Real Fields, Lecture Notes in Math. 1093, Springer, 1984.

[9] P. Ribbenboim, Théorie des Valuations, Les Presses de l'Université de Montréal, 1968.

[10] A. Tarski, The Completeness of Elementary Algebra and Geometry, Hermann, Paris, 1940.

[11] - Contributions to the theory of models I, II, Indag. Math. 16 (1954), 572-588.

Institute of Mathematics Jagiellonian University

Reymonta 4, 30-059 Kraków, Poland

E-mail: nowak@im.uj.edu.pl
Faculteit der Wiskunde en Informatica Vrije Universiteit De Boelelaan 1081a 1081 HV Amsterdam The Netherlands 